\title{
L'EMPLOI DES STANDARDS EN DROIT ROMAIN
}

\section{Elena Giannozzi*}

Key words: Standards; good faith; reasonable man; tort; Roman law; French public law; French private law

Définir en quoi consiste un standard en droit est une question délicate qui relève plus de l'épistémologie que de la science juridique. ${ }^{1}$ En dépit de la difficulté à définir le standard, le droit positif français emploie à plusieurs reprises cette technique, ${ }^{2}$ bien que l'apparition du mot "standard" en droit français soit très récente. Ce terme, qui

1 Voir Bernard 2010: aux pages 19sq.

2 Sur le standard en droit positif, voir Rials 1980: aux pages 1sq. Cet auteur analyse l'apparition du concept de standard en France aux pages 16sq. Un colloque a été consacré au standard en 1988 et il a eu lieu à la Faculté de droit et de Sciences Politiques d'Aix-Marseille. Les actes ont été publiés par la Revue de la recherche juridique, droit prospectif dans le vol 4 de l'année 1988. Parmi les nombreuses contributions, voir notamment: Dale 1988: aux pages 887sq; Delebecque 1988: aux pages 871sq; Delmas-Marty 1988: aux pages 1019sq; Navarro 1988: aux pages 833sq; Orianne 1988: aux pages 1037sq; Pattaro 1988: aux pages 813sq; Petev 1988: aux pages 825sq; Wroblewski 1988: aux pages 847 sq. Il est aussi utile de consulter l'ouvrage collectif dirigé par Perelman \& Vander Elst 1984. Parmi les différentes contributions il faut signaler celle de Carbonnier 1984: aux pages 99sq. Pour le standard en droit communautaire, voir Bernard 2010. Sur le standard à l'aune de l'ordonnance n 2016-131 du 10 février 2016 portant réforme du droit des contrats, du régime générale et de la preuve des obligations en droit français voir Blanc 2016: aux pages 394sq et Sturlèse 2016: aux pages 398sq.

* French Docteur, Université Panthéon-Assas (Paris II). 
dérive du vocabulaire anglais, ${ }^{3}$ apparaît en France au cours de la décennie 1920 dans un mouvement de réaction contre la méthode de l'École de l'Exégèse. ${ }^{4}$

Le renvoi au bon père de famille fut sans doute l'une des utilisations les plus célèbres de la technique du standard. ${ }^{5}$ La réforme du 4 août 2014 l'a effacé au profit des expressions "raisonnable" ou "raisonnablement". Le législateur a maintenu la technique du standard en changeant simplement la référence: la raison se substitue alors au modèle du bon père de famille ${ }^{6}$ qui est considéré comme incompatible avec la parité entre hommes et femmes.

D'aucuns, sans doute influencés par l'emploi récent du mot, ont pu affirmer le caractère contemporain de cette technique. ${ }^{7}$ Pourtant, l'origine romaine du bon père de famille révèle l'ancienneté du mécanisme du standard. ${ }^{8}$ Selon le tenant de cette théorie, la dérivation romaine du bon père de famille ne serait toutefois pas en contradiction avec cette hypothèse car les standards se seraient développés à Rome, à partir notamment de la période antonine et sévérienne. ${ }^{9}$ Le mécanisme du standard semble être considéré comme une sorte de corps étranger qui se serait greffé à l'époque tardive sur le tronc originaire du droit romain. En réalité, cette affirmation ne correspond pas aux données des sources. À titre d'exemple, l'on trouve une figure comparable au modèle du bonus pater familias ${ }^{10}$ dans l'œuvre de Quintus Mucius Scaevola - le célèbre pontife mort en 82 av J-C. Ce jurisconsulte utilise en effet comme modèle l'homme diligent. ${ }^{11}$ L'ancienneté de l'emploi de ce paramètre pour évaluer le comportement d'une personne, dans le cas d'espèce en matière extracontractuelle, ne fait pas de doutes.

3 Un rôle primordial a été joué par la réflexion de Roscoe Pound 1919. L'interprétation du mot standard en anglais et son opposition avec le concept de rule proposée par N Roscoe Pound a engendré des difficultés en doctrine. Elles sont liées à la traduction erronée de rule par règle juridique. Cela a emmené une partie de la doctrine a nier le caractère de règle juridique au standard. À propos de ce débat, voir Bernard 2010: aux pages 6sq.

4 Sur ce point, voir Rials 1980: aux pages 16sq.

5 À ce propos Viney 2013.

6 Sur le reasonable man du droit anglais, voir Dale 1988: aux pages 891sq. Voir aussi MacCormick 1984: aux pages 131 sq.

7 Rials 1980: à la page 30. $C f$ Al-Sanhouri 1925: à la page 29; Stati 1927: aux pages 78sq; Josserand 19392: aux pages 313 sq.

8 Dans ce sens, Ouedraogo 2013: à la page 160 qui situe l'apparition de la technique du standard à l'époque classique. Il faut toutefois remarquer que l'apparition des standards remonte déjà à l'époque préclassique comme le témoigne, par exemple, l'inclusion de la clause ex fide bona dans les formules. Nous retournerons sur cette thématique dans les pages qui viennent.

9 Rials 1980: à la page 30.

10 Le bonus ou diligens pater familias a fait l'objet d'attaques de la part de la doctrine interpolationniste. Notamment, Kunkel 1925: aux pages 266sq qui, plus en général, critique le concept même de diligence. Cette reconstruction a été déjà réfutée par Buckland 1930: aux pages 85sq. La conception hypercriticiste de Kunkel est aujourd'hui abandonnée.

11 Paul 10 Ad Sabinum D 92 31: "Si putator ex arbore ramum cum deiceret vel machinarius hominem praetereuntem occidit, ita tenetur, si is in publicum decidat nec ille proclamavit, ut casus eius evitari possit. Sed Mucius etiam dixit, si in privato idem accidisset, posse de culpa agi: culpam autem esse, quod cum a diligente provideri poterit, non esset provisum aut tum denuntiatum esset, cum periculum evitari non possit. Secundum quam rationem non multum refert, per publicum an 
En revanche, il paraît légitime de s'interroger sur l'opportunité méthodologique d'employer le terme "standard" pour décrire le droit romain. Ne s'agit-il pas d'un anachronisme conceptuel? Il est en effet évident que ce terme anglais ne trouve pas son origine dans les catégories juridiques romaines. Il convient donc de se demander si par l'emploi du concept de "standard" nous ne risquons pas de déformer la réalité $\mathrm{du}$ droit romain pour l'adapter aux catégories contemporaines. Le problème de l'application des notions contemporaines au droit romain a fait couler beaucoup d'encre. L'on peut penser à la querelle, désormais ancienne, qui a opposé Emilio Betti à Pietro De Francisci sur l'opportunité d'employer pour le droit romain le concept de negozio giuridico ou Rechtsgeschäft, qui avait été élaboré par abstraction par la Pandectistique allemande à partir des sources romaines. ${ }^{12}$ Est-il donc légitime de parler de standard par rapport au droit romain alors que la iurisprudentia n'a jamais conceptualisé cette technique?

Il est d'abord nécessaire de donner une définition du standard pour comprendre si elle peut correspondre à ce qui ressort des sources romaines. Cela - nous l'avons déjà souligné - n'est pas une tâche simple. Le standard n'a pas été défini de manière unanime, si bien que cette recherche de définition a pu donner lieu à plusieurs débats doctrinaux. $^{13}$

En dépit de ces importantes discussions sur la nature du standard, on retient la définition proposée par Stéphane Rials puisqu'elle paraît suffisamment neutre. Selon cet auteur, "le standard est un type de disposition indéterminée, plutôt utilisée par le juge, dont le caractère normatif est l'objet des contestations et qui met en jeu certaines valeurs fondamentales de normalité, de moralité ou de rationalité". ${ }^{14}$

per privatum iter fieret, cum plerumque per privata loca volgo iter fiat. Quod si nullum iter erit, dolum dumtaxat praestare debet, ne immittat in eum, quem viderit transeuntem: nam culpa ab eo exigenda non est, cum divinare non potuerit, an per eum locum aliquis transiturus sit." ("Si un élagueur en coupant une branche d'un arbre ou un ouvrier qui travaille sur un échafaudage tue un homme qui passe, il est ainsi responsable, s'il coupe en publique sans le signaler en criant de manière à ce que sa chute puisse être évitée. Mais Mucius [sc Quintus Mucius Scaevola] a dit aussi qu'on pouvait agir pour faute si la même chose arrive sur un terrain privé. En effet, la faute est ce que bien qu'aurait pu être prévu par un homme diligent, n'a pas été prévu ou a été signalé quand désormais le danger ne pouvait plus être évité. Pour cette raison il n'importe pas grande chose si cela arrive dans un terrain public ou privé, puisque souvent on passe couramment dans les terrains privés. Or s'il n’y a pas un chemin, il doit seulement être responsable pour le dol de manière à ce qu'il ne frappe pas celui qu'il voit passer. En effet, la [sc responsabilité pour] faute ne doit pas être exigée de lui car il n'aurait pas pu deviner si quelqu'un allait passér par ce lieu.”)

12 Betti 19972: à la page 75 affirmait qu'il était utile et légitime d'employer le concept de negozio giuridico car il permettait de mieux comprendre, sans pour autant les dénaturer, les sources romaines. Au contraire, De Francisci 1936: aux pages 16sq (=1997² à la page 99) nie l'opportunité du recours à la dogmatique contemporaine. Pour un résumé de la querelle avec bibliographie, voir Scevola 2008: à la page 29 note 20.

13 Bernard 2010: aux pages 6sq.

14 Il s'agit de la définition que Rials 1980: à la page 3 utilise comme point de départ pour son analyse. Il donne une définition aboutie du standard à la page 120: "le standard est une technique de formulation de la règle du droit qui a pour effet une certaine indétermination a priori de celleci. Souvent d'origine jurisprudentielle, et en principe dénoté par l'utilisation de certaines formes, le standard vise à permettre la mesure de comportements et de situations en termes de normalité, dans la double acception de ce terme." 
Cette définition, exception faite pour le passage relatif aux contestations sur le caractère normatif du standard, s'adapte aux données qui découlent des sources romaines, ${ }^{15}$ comme c'est le cas du fragment d'Ulpien 30 Ad edictum D 13722 4:

Si creditor, cum venderet pignus, duplam promisit (nam usu hoc evenerat et conventus ob evictionem erat et condemnatus), an haberet regressum pigneraticiae contrariae actionis? Et potest dici esse regressum, si modo sine dolo et culpa sic vendidit et ut pater familias diligens id gessit: si vero nullum emolumentum talis venditio attulit, sed tanti venderet, quanto vendere potuit, etiamsi haec non promisit, regressum non habere. ${ }^{16}$

Ulpien examine le cas d'un créancier qui a vendu la chose gagée, ${ }^{17}$ après s'être engagé avec l'acheteur par une stipulatio dupla en cas d'éviction. Celle-ci a effectivement lieu: le créancier peut-il se retourner contre son débiteur par le biais de l'actio pigneraticia contraria? Le juriste sévérien répond de manière affirmative si le créancier s'est comporté sans dol, sans faute et comme l'aurait fait un père de famille diligent. Le prudens pater familias coïncide avec un standard, à savoir cette disposition indéterminée, qui met en œuvre certaines valeurs fondamentales de normalité, de moralité ou de rationalité. En effet, le père de famille prudent et diligent n'est rien d'autre qu'un modèle qui correspond à l'idée de comportement normal propre aux Romains. Grâce à son caractère indéterminé, le juge pourra apprécier les circonstances factuelles et décider si le vendeur s'est comporté d'une manière qui est conforme au modèle qu'il doit appliquer.

L'on relève d'autres exemples chez les juristes classiques, comme le fragment de Julien 2 Ad Urseium Ferocem D 45161 relatif aux boni mores: "Stipulatio hoc modo concepta: 'si heredem me non feceris, tantum dare spondes' inutilis est, quia contra bonos mores est haec stipulatio." 18

Tout comme le bonus ou le diligens pater familias, les bonnes mœurs sont un standard, un modèle qui trouve donc son fondement dans les valeurs profondes de la société et qui permet aux juges d'opérer un contrôle sur le contenu d'un acte juridique, en l'occurrence une stipulation.

15 Dans ce sens, voir aussi Delebecque 1988: à la page 873.

16 "Si le créancier, en vendant le gage, s'est engagé par une stipulation au double (en effet, cette éventualité s'est produite et il avait été assigné en justice pour éviction et condamné), peut-il se retourner $<$ contre son débiteur $>$ par l'action contraire du gage? Et il est possible de dire qu'il peut se retourner <contre son débiteur>, si seulement il a effectué la vente sans dol et sans faute et il s'est comporté comme un diligent père de famille. En revanche, si une telle vente n'a apporté aucun gain, mais il l'aurait vendu au prix auquel il aurait pu le vendre, même sans pronettre cela, il n'a pas de retour."

17 Voci 1990: à la page 86 note 94 observe que le texte se référait probablement à l'origine à la fiducia. À son avis, la référence au diligens pater familias constitue une interpolation pour mettre en adéquation le fragment d'Ulpien avec le nouveau contexte du gage. Néanmoins, il ne semble pas qu'il ait d'éléments suffisants pour prouver cet ajout. MacCormack 1972: aux pages 132, 165; et Cannata 1992: à la page 29 note 95 ne mentionnent pas d'interpolations.

18 "Une stipulation formulée de cette manière: 'si tu ne me nommes pas héritier, promets-tu de donner tant' est inutile puisque cette stipulation est contraire aux bonnes mœurs." 
La catégorie du standard, tout en étant conceptualisée à une époque récente, trouve donc une correspondance en droit romain et semble pouvoir être appliquée pour l'étudier. L'on peut néanmoins se demander quelle est l'utilité d'employer ce terme, entièrement anachronique, pour décrire la réalité romaine. Or, il semble qu'une telle application se révèle profitable puisque elle permet de pallier l'absence de théorisation de la jurisprudence romaine. Le manque d'un terme latin qu'indique le standard peut cacher le fait que les juristes et le préteur avaient recours à des expressions qui répondaient toutes à une finalité analogue, à savoir d'attribuer au juge une marge d'appréciation plus large. En utilisant la catégorie du standard, l'on dispose d'un instrument qui permet de décrire de manière synthétique plusieurs expressions qui sont présentes dans les sources en cernant ainsi leur finalité qui est l'élargissement des pouvoirs du juge. L'emploi du mot standard dans l'étude du droit romain peut dès lors constituer un outil pour mieux comprendre les pouvoirs du juge et sa marge de discrétion dans l'interprétation des actes juridiques.

L'objectif de ce travail n'est pas de faire un catalogue systématique de différentes expressions que l'on peut assimiler aux standards en analysant les sources, mais plutôt de montrer le fonctionnement de cette catégorie juridique et les raisons qui ont amené les Romains à l'employer. En effet, tout comme en droit positif, l'on peut repérer dans les sources romaines plusieurs standards. L'on peut citer comme exemples le bonus ou diligens pater familias, les boni mores ou encore la fides bona, le vir bonus et le bonum et aequum. Or, il est possible de reconduire à une bipartition cette liste qu'à première vue peut paraître aléatoire. Tout standard fonctionne par la référence à un modèle qui, en reprenant l'expression de S Rials, "met en jeu certaines valeurs fondamentales de normalité, de moralité ou de rationalité". ${ }^{19} \mathrm{~L}$ 'on remarque alors l'existence de deux typologies de modèles de référence auquel ces différents standards se rattachent. Dans un premier cas le standard se réfère à un concept abstrait comme la bonne foi, l'équité ou les bonnes mœurs. En revanche, dans la seconde hypothèse, il est incarné par un individu qui constitue un modèle de conduite. C'est le cas de l'homme de bien et du bon ou diligent père de famille. L'on peut dès lors parler de standard conceptuel (1) et de standard incarné (2) selon le différent modèle de référence. Cette bipartition permet de trouver un fil conducteur dans l'étude des exemples de standard que l'on trouve dans les sources romaines.

\section{Le standard conceptuel}

Le standard que l'on a défini comme conceptuel a pour modèle de référence une idée abstraite. L'on peut rattacher à cette catégorie la fides bona, le bonum et aequum et les boni mores. 


\section{La fides bona}

En droit contemporain, la bonne foi ne peut être considérée, de manière générale, comme un standard. Elle ne pourrait en effet être ainsi qualifiée seulement dans une acception très particulière, à savoir la bonne foi objective..$^{20}$ Cette affirmation trouve un écho en droit romain qui envisage aussi la fides bona sous un double angle, objectif et subjectif. ${ }^{21}$

Dans son sens objectif, la bonne foi consiste dans un modèle sur la base duquel le juge doit apprécier le comportement des cocontractants et qui trouve son fondement dans la clause ex fide bona. Elle est insérée dans la formule de certaines actions qui sont ainsi dénommées les actions de bonne foi.

La procédure formulaire romaine se caractérise en effet par une opposition entre les actions de droit strict et de bonne foi. ${ }^{22}$ On ne dispose pas d'éléments précis qui permettent de dater avec certitude le moment de la création des formules de bonne foi, situées entre le $\mathrm{III}^{\mathrm{e}}$ et le $\mathrm{II}^{\mathrm{e}}$ siècle av $\mathrm{J}-\mathrm{C} .{ }^{23}$ L'origine des actions de bonne foi est dès lors très controversée en doctrine. En revanche, l'analyse de leur structure permet de comprendre la raison de leur création : l'extension des pouvoirs du juge.

Dans le cas des actions de droit strict, le iudex est limité dans son pouvoir d'évaluation car il est lié à la lettre de la formula. ${ }^{24} \mathrm{Il}$ en va différemment pour les actions de bonne foi car la référence à la bonne foi est insérée dans leurs formules. L'inclusion de la formule ex fide bona permet donc d'élargir la faculté d'appréciation du iudex qui peut désormais prendre en compte de manière globale le comportement des parties, comme le montre le célèbre passage du De officiis 370 de Cicéron: ${ }^{25}$

Nam quanti verba illa: UTI NE PROPTER TE FIDEMVE TUAM CAPTUS FRAUDATUSVE SIM! Quam illa aurea: UT INTER BONOS BENE AGIER OPORTET ET SINE FRAUDATIONE! Sed, qui sint "boni”, et quid sit "bene agi”, magna quaestio est. Q quidem Scaevola, pontifex maximus, summam vim ${ }^{26}$ esse dicebat in omnibus is arbitriis, in quibus adderetur EX FIDE BONA, fideique bonae nomen existimabat manare latissime, idque versari in tutelis, societatibus, fiduciis, mandatis, rebus emptis, venditis, conductis,

20 Idem à la page 102.

21 Sur la vexata quaestio de la signification originaire de la bona fides voir avec bibliographie l'article de Fiori 2011a: aux pages 99sq.

22 Les romanistes ont discuté avec ardeur sur l'origine prétorienne ou civile des actions de bonne foi. Dans le passé la doctrine était plutôt en la faveur d'une origine prétorienne, aujourd'hui cela a été remis en question. Voir en ce sens: Fiori 1998-1999: aux pages 165sq; idem 2008: aux pages 248 sq.

23 La mort en 82 av J-C de Quintus Mucius Scaevola le pontife, qui connaît les actions de bonne foi, constitue un terminus ante quem.

24 On verra toutefois comment par l'emploi du standard du vir bonus, le juge obtient une plus large latitude d'appréciation dans le domaine des stipulations prétoriennes.

25 La bibliographie sur ce passage est immense. Voir les contributions récentes de Noordaven 1999: aux pages 302sq; Dunand 2000: aux pages 174sq; Cardilli 2010²: aux pages 29sq; Naumowicz 2011: aux pages 109sq; Bertoldi 2012: aux pages 25sq.

26 Sur les interprétations données à summa vis, voir Cardilli 2010²: à la page 35 note 65 . 
locatis, quibus vitae societas ${ }^{27}$ contineretur; in iis magni esse iudicis statuere, praesertim cum in plerisque essent iudicia contraria, quid quemque cuique praestare oportere. ${ }^{28}$

L'Arpinate relate dans ce passage la pensée de Quintus Mucius Scaevola. Il souligne l'importance de la fides bona et d'une catégorie plus générale, le bonae fidei nomen, qui regroupe un groupe d'actions analogues aux actiones ex fide bona, comme la fiducia. ${ }^{29} \mathrm{La}$ formule de cette action inclut en effet une référence à un standard analogue à la bonne foi, l'ut inter bonos bene agier. Les arbitria bonae fidei sont caractérisés par une grande force (magna vis) et le concept de bonne foi (fidei bonae nomen) $)^{30}$ a une extension considérable (manare latissime) puisqu'il caractérise la tutelle, la société, le mandat, la vente et le louage. Le juge a un rôle essentiel pour déterminer les obligations des parties. ${ }^{31}$

L'insertion de la clause ex fide bona revêt une importance pratique remarquable. Quelques cas tirés de la jurisprudence permettent d'apprécier le rôle joué par la

27 Selon Naumowicz 2011: aux pages 102sq l'expression societas vitae doit être interprétée comme une notion d' "affinité sociale". Sur cette expression, voir avec bibliographie Falcone 2013b: 269sq.

28 'Mais nous n'avons aucune représentation solide et claire du vrai droit et de la pure justice, nous nous servons d'une image et des échos. Ah, si on suivait au moins celles-ci! En effet, elles sont révélées par des excellents exemples de la nature et de la vérité. Quelle importance ont ces célèbres mots: 'afin que je ne sois pas ni abusé ni trompé frauduleusement par toi et ta promesse' ou cette merveilleuse formule: 'ainsi qu'il convient agir parmi des hommes de bien'! Mais la grande question est de savoir qui sont les 'bons' et ce qu'est 'agir bien'. Q Scaevola, le grand pontife, disait qu'il y avait plus de force dans les jugements, dans lesquels il était ajouté 'sur la base de la bonne foi'. Il estimait que le concept de bonne foi a une très grande extension et qu'on le trouve dans les tutelles, les sociétés, les fiducies, les mandats, dans les affaires d'achat et de vente, de prise de bail et de location, actes qui impliquent une société de vie; dans ces contrats c'est un grand juge qui doit décider, notamment parce que dans la plupart des cas il y a des jugements contraires, ce que chacun doit à l'autre."

29 Cette affinité forte qui est perçue entre la fiducia et les actions de bonne foi débouchera sur son inclusion dans cette catégorie ainsi qu'il est montré par Gaius 4 62. Tout comme l'ut inter bonos bene agier de la fiducia, un autre standard analogue à la bonne foi est inséré dans la formule de l'actio rei uxoriae. Il s'agit de l'aequius melius qui est rappelé par Cicéron, Topica 17 66: "In omnibus igitur eis iudiciis, in quibus ex fide bona est additum, ubi vero etiam ut inter bonos bene agier oportet, in primisque in arbitrio rei uxoriae, in quo est quod eius aequius melius, parati eis esse debent. Illi dolum malum, illi fidem bonam, illi aequum bonum, illi quid socium socio, quid eum qui negotia aliena curasset ei cuius ea negotia fuissent, quid eum qui mandasset, eumve cui mandatum esset, alterum alteri praestare oporteret, quid virum uxori, quid uxorem viro tradiderunt" ("Dans tous ces jugements dans lesquels il est ajouté "sur la base de la bonne foi", ou aussi dans ceux "ainsi qu'il faut agir bien parmi des hommes de bien", et avant tout dans le jugement arbitral sur le patrimoine de la femme, dans lequel il faut suivre ce qui est le meilleur et le plus équitable, les jurisconsultes doivent être prêts [ $s c$ avec leur conseil]. Ils ont établi en quoi consiste le dol, la bonne foi, le bon et l'équitable, les obligations réciproques des associés, de celui qui gère les affaires d'autrui envers la personne à qui sont les affaires, du mari à la femme, de la femme au mari.")

$30 \quad C f$ Naumowicz 2011: aux pages 96sq.

31 Behrends 2002: à la page 218. 
bonne foi dans l'interprétation des actes juridiques. On peut citer, à titre d'exemple, le fragment de Labéon 4 Posteriores a Iavoleno epitomati D 19 1 50:32

\begin{abstract}
Bona fides non patitur, ut, cum emptor alicuius legis beneficio pecuniam rei venditae debere desisset antequam res ei tradatur, venditor tradere compelletur et re sua careret. Possessione autem tradita futurum est, ut rem venditor aeque amitteret, utpote cum petenti eam rem $<$ emptor exceptionem rei venditae et traditae opponere possit nec perinde sit, quasi eam rem $>33$ petitor ei neque vendidisset neque tradidisset. ${ }^{34}$
\end{abstract}

Le juriste augustéen se fonde sur le standard de la bonne foi pour nier que le vendeur soit obligé de remettre la chose vendue alors que l'acheteur, grâce à une certaine loi, n'est plus obligé de payer le prix. Les conséquences de l'application de la fides bona sont donc extrêmement importantes car par ce biais le juriste parvient à paralyser l'obligation du vendeur de remettre la chose. On peut même affirmer qu'en interprétant le contrat par le standard de la bonne foi, l'obligation de rem tradere n'existe pas: à cause de la promulgation de la loi, il n'y a plus un oportere ex fide bona à la charge du venditor ${ }^{35}$ En revanche, si la possession de la chose a été transférée, le vendeur ne peut pas la revendiquer. Une telle solution n'est pas contraire à la bonne foi. En effet, Labéon observe que la revendication du vendeur ne pourrait pas aboutir car l'acheteur pourrait toujours lui opposer à bon droit l'exceptio rei venditae et traditae.

Un passage de Scaevola 2 Responsa D 19148 renferme une autre illustration de l'application du standard de la bonne foi:

Titius heres Sempronii fundum Septicio vendidit ita: "fundus Sempronianus, quidquid Sempronii iuris fuit, erit tibi emptus tot nummis" vacuamque possessionem tradidit neque fines eius demonstravit: quaeritur, an empti iudicio cogendus sit ostendere ex instrumentis hereditariis, quid iuris defunctus habuerit et fines ostendere. Respondi id ex ea scriptura

32 Il s'agit d'un texte très célèbre, sur lequel il existe une bibliographie extrêmement vaste. Voir praecipue: Talamanca 1997: aux pages 353sq; Gallo 2000: aux pages 1sq. Cf avec bibliographie Fiori 2011a: aux pages 161sq.

33 La version du texte relatée par les manuscrits "utpote cum petenti eam rem petitor ei neque vendidisset neque tradidisset" est incompréhensible. Nous suivons l'intégration proposée par Mommsen, ad $h \mathrm{l}$. Dans ce sens Talamanca 1997: aux pages 372sq et de manière hypothétique Fiori 2011a: à la page 162 note 189. Cette reconstruction n'est pas accueillie par Gallo 2000: aux pages 1 sq. L'auteur nie qu'on puisse attribuer à aeque la signification d' "également" en privilégiant celle "de manière équitable".

34 "La bonne foi ne tolère pas que le vendeur soit obligé de transmettre sa chose et qu'il en soit privé quand l'acheteur a cessé de devoir l'argent pour la chose vendue grâce à une certaine loi avant que la chose ne lui soit remise. En revanche, si la possession a été transmise il arrivera que le vendeur perdrait également sa chose vu que l'acheteur pourrait opposer à celui-ci, qui réclame sa chose, l'exception de la chose vendue et transmise et il ne serait pas comme si le demandeur ne lui avait pas vendu ou transmis cette chose."

35 Fiori 2011a: à la page 164. 
praestandum, quod sensisse intelleguntur: quod si non appareat, debere venditorem et instrumenta fundi et fines ostendere: hoc etenim contractui bonae fidei consonat. ${ }^{36}$

La question qui est posée à Scaevola porte sur le point de savoir quelles obligations s'imposent au vendeur sur la base d'un contrat de vente qui a comme objet un fonds reçu en héritage. En effet, l'héritier désigne de manière vague-fundus Sempronianus, quidquid Sempronius iuris fuit - l'objet de la vente. De surcroît, il ne montre pas quelles sont les bornes. ${ }^{37} \mathrm{Il}$ y a donc une ambiguïté sur la définition même de la chose vendue. Est-ce que cette incertitude implique que le vendeur doit révéler les documents relatifs au fonds vendu et montrer à l'acheteur les limites du domaine? La solution qui est proposée par Scaevola trouve son fondement dans la nature de bonne foi de l'emptio venditio. La fides bona guide le juriste dans l'interprétation du contrat: le vendeur sera contraint à instrumenta fundi et fines ostendere si la teneur du testament ne montre pas en quoi consiste le fonds à vendre.

Un mécanisme analogue à la fides bona, comme le montre son assimilation aux actions de bonne foi, ${ }^{38}$ est représenté par la clause quod eius melius aequius erit qui est insérée dans la formule de l'actio rei uxoriae. ${ }^{39}$ Dans ce cas, le renvoi au bonum et aequum peut être considéré comme un standard.

\section{Le bonum et aequum}

La clause quod eius melius aequius erit présente des éléments d'analogie avec la bona fides. Une différence importante doit cependant être soulignée. Dans les actions de bonne foi, la clause ex fide bona apparaît dans l'intentio de l'action, alors que la clause quod eius melius aequius erit est utilisée dans la condemnatio. Cela signifie que la bonne foi touche l'ensemble de l'acte juridique entre les parties, alors que le melius aequius ne concerne que le moment de la condamnation.

En cas de dissolution du mariage, la femme sui $i u r i s^{40}$ ou son père si elle est décédée, pour la dos profecticia, ${ }^{41}$ peut agir pour obtenir la restitution de la dot.

36 "Titius l'héritier de Sempronius a vendu le fonds à Septicius de cette manière: 'Tu achèteras pour une certaine somme le fonds Sempronianus, tout ce qui était du droit de Sempronius' et il a transmis la possession vacante et il n'a pas montré les limites de la propriété. Il est demandé si par le jugement d'achat $<$ le vendeur $>$ doit être obligé à montrer sur la base des documents relatifs à l'héritage ce sur quoi le défunt avait droit et montrer les limites. Il [sc Scaevola] a répondu qu'il faut donner sur la base de l'acte ce qu'ils avaient entendu; mais s'il n'apparaît pas, le vendeur doit montrer les documents relatifs au fonds et les limites. En effet, cela est en harmonie avec un contrat de bonne foi."

37 Voci 1987: à la page 115 note 72 observe que le verbe "demonstrare" peut être considéré comme le terme technique en matière de traditio.

38 Gai 462.

39 Sur les problèmes de la reconstruction de la formule de l'actio rei uxoriae, voir Varvaro 2006.

$40 \mathrm{Si}$ la femme est alieni iuris, l'action en justice revient à son pater familias. Toutefois, l'accord de la femme est nécessaire (Tituli ex corpore Ulpiani 6 6).

41 La dos profecticia est celle qui a été constituée par le pater familias de la femme (Tit ex corp Ulp 6 $3)$. 
Puisque la formule de l'actio rei uxoriae mentionne le quod eius melius aequius erit, le juge n'est pas obligé de condamner le mari à restituer à la femme ou à son père l'intégralité de la dot. ${ }^{42}$ Ainsi, l'époux peut obtenir le beneficium competentiae. ${ }^{43}$ En outre, le juge peut disposer des retenues en sa faveur, par exemple, s'il y a des enfants ou si la femme a eu une mauvaise conduite au cours du mariage. ${ }^{44}$ Quelques passages des Tituli ex corpore Ulpiani attestent que dans ces hypothèses il existe des règles jurisprudentielles qui guident l'activité du juge et qui permettent de prévoir de manière forfaitaire la retenue à opérer sur la dot. ${ }^{45}$

Le mari qui a dépensé de l'argent pour l'entretien des biens dotaux, aura droit à une retenue à cause d'impenses si ces frais sont utiles. ${ }^{46} \mathrm{Si}$ les impenses sont nécessaires, ${ }^{47}$ elles sont déduites de plein droit de la dot. ${ }^{48}$ Il revient alors au iudex sur la base de la clause quod melius aequius erit de calculer la dot une fois évaluées les impenses. ${ }^{49} \mathrm{Il}$ n'est dès lors pas étonnant que le titre du Digeste 251 soit consacré à l'analyse des différentes hypothèses d'impenses, ce qui permet de guider le iudex dans sa tâche.

La formule de l'actio rei uxoriae ne constitue pas un cas isolé car elle fait partie du groupe plus large des actiones in bonum et aequum conceptae..$^{50} \mathrm{Ce}$ groupe d'actions, auquel appartiennent, par exemple, l'actio iniuriarium ou l'actio sepulchri violati, a fait l'objet des nombreux débats en doctrine.$^{51}$ Les controverses portent par exemple sur le point de savoir si la formulation originaire de ces actions mentionnait le bonum et aequum ou seulement le aequum. De surcroît, les auteurs ne sont pas unanimes sur l'identification des actions qui à l'époque classique appartenaient au

42 En outre, le juge peut disposer en la faveur du mari des dilations dans les paiements.

43 Le beneficium competentiae signifie que le mari est condamné à restituer la dot en proportion avec ses capacités économiques. Sur le beneficium competentiae, voir De Loynes de Fumichon 2012: aux pages 495-522.

44 Tit ex corp Ulp 6 9: Retentiones ex dote fiunt aut propter liberos, aut propter mores, aut propter inpensas, aut propter res donatas, aut propter res amotas. ("Les retenues de dot se font ou à cause des enfants ou à cause des mœurs ou à cause des dépenses ou à cause des choses qui ont été volées ou à cause des choses qui ont été détournées.")

45 Tit ex corp Ulp 6 4; $610 ; 612$.

46 Tit ex corp Ulp 6 16: Utiles sunt, quibus non factis quidem deterior dos non fuerit, factis autem fructuosior effecta est, veluti si vineta et oliveta fecerit. ("Les impenses sont utiles quand, si elles ne sont pas faites, la dot ne sera pas ruinée, mais si elles sont faites la dot devient plus rentable, comme si des vignes ou des oliveries sont créées.")

47 Tit ex corp Ulp 6 15: Necessariae sunt inpensae, quibus non factis dos deterior futura est, veluti si quis ruinosas aedes refecerit. ("Les impenses sont nécessaires quand, si elles ne sont pas faites, la dot sera ruinée, comme par exemple si quelqu'un refait un bâtiment qui tombe en ruine.”)

48 Cf Ulp 36 ad Sab D $2515 p r$.

49 Lévy 1937: aux pages 102-103.

50 L'expression actiones in bonum et aequum conceptae est employée une seule fois par Papinien 8 quaest D 4712 10. Ulpien 57 ad ed D 4710111 y fait allusion de manière indirecte: iniuriarium actio ex bono et aequo est. Cf Paricio: 1986 à la page 7. Cet auteur, aux pages 97sq, nier l'appartenance de l'actio rei uxoriae au catalogue des actiones in bonum et aequum conceptae.

51 Voir avec bibliographie: Paricio 1986. 
groupe des actiones in bonum et aequum conceptae. ${ }^{52}$ En revanche, la fonction de la condamnation in quantum aequum videbitur ne soulève pas de difficultés. Il apparaît en effet que, tout comme dans l'actio rei uxoriae, le but du renvoi au bonum et aequum est toujours d'attribuer une plus grande marge de discrétion au juge. ${ }^{53} \mathrm{Un}$ passage du livre 45 Ad edictum de Paul au Digeste 471016 en matière d'actio iniuriarum confirme clairement notre propos: "Sed non esse aequum pro maiore parte, quam pro qua dominus est, damnationem fieri Pedius ait: et ideo officio iudicis portiones aestimandae erunt." ${ }^{4}$

Le passage porte sur une hypothèse d'iniuria au détriment d'un esclave qui a plusieurs propriétaires. Le jurisconsulte Pedius, cité par Paul, affirme qu'il serait contraire à l'aequum de condamner le reus de manière disproportionnée à sa quotepart sur l'esclave. En revanche, la solution qui est conforme à l'aequum est de laisser le juge calculer les dommages et intérêts. L'inclusion de l'aequum dans la formule de l'actio iniuriarum augmente de manière manifeste les pouvoirs discrétionnaires du iudex. Une telle donnée ne peut être remise en question.

En revanche, l'interprétation qu'il convient de donner à la clause in bonum et aequum soulève une difficulté. Il en va de même de son rapport avec le concept d'aequitas ${ }^{55} \mathrm{Il}$ s'agit d'un des problèmes les plus épineux de la doctrine romanistique. Et, à bien des égards, ces nombreuses dissensions justifient l'existence d'une littérature extrêmement vaste. ${ }^{56}$ Pour quelle raison les jurisconsultes ont recours à l'aequitas pour l'interprétation des actes juridiques même en dehors du domaine des actions in bonum et aequum conceptae?

De manière générale, on peut considérer que le bonum et aequum est plus ancien que le renvoi à l'aequitas. En effet, alors que la première expression se trouve déjà dans le théâtre de Plaute, ${ }^{57}$ la deuxième - un substantif abstrait - porte la marque d'une pensée postérieure, qui remonte probablement au I ${ }^{\mathrm{er}}$ siècle av J-C. ${ }^{58} \mathrm{Il}$ est donc envisageable que le recours à l'aequitas implique une certaine généralisation du bonum et aequum, ce qui lui confère un caractère plus abstrait dans la réflexion des jurisconsultes. Un tel phénomène ne peut qu'expliquer la raison pour laquelle l'aequitas n'apparaît plus exclusivement dans les actiones in bonum et aequum conceptae. Un témoignage pertinent est offert par un fragment du livre 3 Ad edictum

52 Paricio 1986: à la page 10.

53 Il faut néanmoins observer qu'il y a eu des controverses sur le point de savoir comment interpréter cette marge de manœuvre accrue du juge. $C f$ Paricio 1986: à la page 13.

54 "Mais Pedius affirme qu'il n'est pas équitable que la condamnation soit faite pour une partie plus grande par rapport à celle dont il [sc chaque demandeur] est propriétaire. Et donc il appartiendra à l'office du juge d'estimer les parties."

55 Cette expression a fait l'objet des critiques de la part de la doctrine interpolationniste. $C f$ Beseler 1925: aux pages 453 sq.

56 Voir Pringsheim 1932: aux pages 78sq; Guarino 1960: aux pages 619sq; Maschi 1962: aux pages 428sq; Biscardi 1973: aux pages 137sq.

57 Pl Men v 580.

58 Cf Cic Top 28, 31, 90-91. 
provinciale de Gaius qui est conservé au Digeste 3346 6: "Litis impendia bona fide facta vel ab actoris procuratore vel a rei debere ei restitui aequitas suadet." ${ }^{9}$

Il est question des dépenses qui ont été faites de bonne foi par le procurateur. Si la bonne foi dont il est question doit être ici entendue en sens subjectif, il en va différemment pour l'aequitas. En effet, c'est sur cette base que le jurisconsulte motive son opinion alors qu'ici il ne s'agit pas d'une actio in bonum et aequum concepta. Il convient toutefois de nuancer l'analyse en considérant que la relation entre la partie au procès et le procurator est sanctionnée par une action de bonne foi. ${ }^{60} \mathrm{Il}$ est alors envisageable que le renvoi à l'équité trouve son fondement dans la nature de bonne foi de l'action. ${ }^{61}$

Toutefois, le fragment du livre 8 des Disputationes d'Ulpien 8 au Digeste 5226 révèle le recours à l'aequum dans une matière en dehors du domaine de la bonne foi, comme celle de l'institution d'héritier: "Si sub hac condicione fuerit heres institutus 'si Stichum manumiserit' et manumisisset, et posteaquam manumisit inofficiosum vel iniustum testamentum pronuntietur: aequum est huic quoque succurri, ut servi pretium a manumisso accipiat, ne frustra servum perdat." ${ }^{\prime 62}$ C'est donc l'aequum qui fonde la décision d'Ulpien. Dans plusieurs passages du Digeste il apparaît de manière claire que c'est sur cette base ou sur celle de l'aequitas que les jurisconsultes résolvent plusieurs questions. Souvent c'est même le préteur qui décide car une telle solution est aequissima ou car elle est imposée par l'aequitas. L'on peut dès lors lire des phrases comme aequissimun praetori visum est ${ }^{63}$ ou hoc edictum praetor naturalem aequitatem secutus proposuit. ${ }^{64}$

Il devient alors légitime de se demander s'il est correct de continuer à qualifier de standard l'équité quand elle devient le fondement, la ratio qui justifie une solution de la jurisprudence ou du préteur. Certes, l'équité met toujours en jeu les valeurs de normalité et d'honnêteté dans un système juridique. Toutefois, ses contours semblent s'estomper et, par conséquent, deviennent plus amples. L'aequum bonum n'est plus l'outil qui permet d'élargir les pouvoirs discrétionnaires du juge, mais il devient un instrument plus large de politique juridique dont se servent les prudentes, le praetor

59 "L'équité conseille que les dépenses pour le litige qui ont été faites en bonne foi par le procurateur du demandeur ou du défendeur lui doivent être restituées."

60 En matière d'actions de bonne foi, on peut citer par exemple, Ulp 10 ad ed D 3539 ou Id, 16 ad ed D $6117 p r$.

61 La fonction du procurator ad litem trouve son origine dans le iussum ou mandatum du dominus litis. L'actio mandati, tout comme l'actio negotiorum gestio, est caractérisée par le renvoi à la bonne foi. $C f$ Gaius 4 84. Sur le procurator ad litem et sur son caractère classique, voir Miceli 2008: aux pages 219 sq.

62 "L'héritier a été institué sous cette condition qu'il affranchisse Stichus et il l'a affranchi, mais après l'affranchissement le testament est prononcé inofficieux ou injuste. Il est équitable de lui venir aussi en aide de manière à ce qu'il reçoive le prix de l'esclave par l'affranchi afin qu'il ne perde pas l'esclave en vain."

63 Ulp 79 ad ed D $791 \mathrm{pr}$.

64 Ulp 11 ad ed D $441 p r$. 
et même l'empereur. ${ }^{65}$ Dans ce sens, il semble être analogue au recours à l'humanitas et à la caritas qui caractérise les décisions impériales d'époque sévérienne. ${ }^{66}$ En effet, l'importance du bonum et aequum dans la réflexion de la jurisprudence classique est bien représentée par la célèbre définition de Celse du droit comme ars boni et aequi, relatée par Ulpien dans un passage du Digeste qui suscité d'innombrables commentaires. ${ }^{67}$

La technique du standard met en jeu des valeurs essentielles d'une société donnée. Or, à Rome les boni mores en montrent une claire exemplification de l'emploi des standards.

\section{Les boni mores}

Le terme "mos" désigne la coutume ou l'usage. L'expression "mos maiorum" renvoie à la coutume des ancêtres, à savoir l'ensemble des traditions et des comportements qui constituaient le fondement même de la société romaine. Papinien dans un extrait du livre 16 des Quaestiones qui est conservé au Digeste 28715 affirme dès lors que "quae facta laedunt pietatem existimationem verecundiam nostram, et generaliter dixerim, contra bonos mores fiunt, nec facere nos posse credendum". ${ }^{68}$

Sur la base des boni mores les jurisconsultes considèrent dès lors qu'il est possible d'apprécier le contenu des obligations, comme c'était le cas pour la stipulation dans le texte de Julien 2 Ad Urseium Ferocem D 451 61. Un autre exemple analogue dans le domaine testamentaire est rapporté par Paul dans son livre 45 Ad edictum qui a été transmis au Digeste 287 9: "Condiciones, quae contra bonos mores inseruntur, remittendae sunt, veluti 'si ab hostibus patrem suum non redemerit', 'si parentibus suis patronove alimenta non praestiterit"”. ${ }^{69}$

Le fragment de Paul donne quelques exemples de comportements jugés contraires aux bonnes mœurs, comme le fait de ne pas racheter le père qui est prisonnier chez les ennemis ou de ne pas prêter les aliments aux parents ou au patron. L'inclusion de ces clauses dans les dispositions mortis causa ne les rend pas nulles, ${ }^{70}$ à la différence des stipulations. Une telle faveur s'explique par le principe du favor testamenti qui conduit les jurisconsultes à interpréter les actes de dernière volonté de manière à en garantir l'application. En effet, il est toujours possible de conclure une nouvelle

65 Par exemple: C I 1262 (Alex Sev année 235); C I 213 (Sept Sev et Car année 202); C I 214 (Car année 212).

66 À ce propos, voir Palma 1992: passim.

67 Ulp 1 instit D $111 \mathrm{pr}$. Sur ce texte extrêmement connu, voir avec la bibliographie Mantello 2007: à la page 121 note 3 .

68 "Il faut croire que nous ne pouvons pas accomplir les faits qui lèsent notre piété, réputation ou pudeur et, comme nous dirions de manière générale, qui sont accomplis contre les bonnes mœurs."

69 "Les conditions qui sont insérées contre les bonnes mœurs doivent être abandonnées comme 'si tu n'as pas racheté ton père des ennemis' ou 'si tu n'as pas donné à tes parents ou au patron les aliments'."

70 À ce propos, voir Voci 1963: aux pages 610sq. 
stipulation alors qu'un acte mortis causa ne pourra être modifié après la mort du disposant.

Il convient de remarquer que le standard des boni mores peut être employé avec celui la fides bona, ainsi que le montre le fragment du livre 28 des Quaestiones de Papinien au Digeste 221 5: "Generaliter observari convenit bonae fidei iudicium non recipere praestationem, quae contra bonos mores desideretur." ${ }^{\prime 11}$

Un contrat qui est protégé par une action de bonne foi est donc incompatible avec une prestation qui est contraire aux bonnes mœurs. La lecture des passages relatifs aux boni mores révèle alors l'ample application du standard qui n'est donc pas exclusivement limité aux actes juridiques de droit strict qui sont dépourvus de l'inclusion dans la formule du critère herméneutique de la bonne foi.

L'analyse des exemples de standard conceptuel a révélé tout l'intérêt d'appliquer au droit romain cette catégorie, qui pourtant est anachronique. En analysant à l'aune du standard des concepts comme la bonne foi, l'équité ou les bonnes mœurs, l'on peut s'apercevoir des similarités qui les rapprochent au-delà des différences. Le lien qui unit ces trois clauses est constitué par l'élargissement des pouvoirs du juge. L'utilité des standards, hier comme aujourd'hui, consiste dans l'augmentation de la marge de discrétion dont jouit le juge. En droit romain, cet élargissement se fait au premier chef par l'insertion des clauses comme la fides bona ou le bonum et aequum dans la formule de l'action. Cette solution est très importante, comme l'atteste l'exemple de la bonne foi. Dans certaines hypothèses cependant l'augmentation des pouvoirs d'appréciation du juge est obtenue en dehors de la formule ainsi que le révèlent certains emplois du bonum et aequum ou les boni mores.

Dans les sources romaines le standard n'est pas seulement présent en tant que concept, mais il peut parfois être incarné par un modèle idéal d'individu. L'analyse des sources révélera que le standard incarné a des caractéristiques identiques, au-delà du modèle de référence, au standard conceptuel. En effet, ces standards, qu'ils soient insérés ou pas dans la formule de l'action ont aussi comme finalité l'élargissement de la marge d'appréciation du juge.

\section{Le standard incarné}

À la lecture des textes romains, l'on repère aisément deux concepts que l'on peut rattacher à la catégorie du standard incarné: le vir bonus et le diligens ou bonus pater familias. En dépit de leur proximité sémantique, il est nécessaire de distinguer ces deux concepts.

\section{Le vir bonus}

Dans les sources juridiques romaines, il y a plusieurs dizaines d'occurrences du vir bonus. Seulement dans le Digeste, nous en trouvons quatre-vingt-quatre. L'homme

71 "Il convient qu'il soit observé de manière générale que le jugement de bonne foi n'accepte pas le prestation qui est demandée contre les bonnes mœurs." 
de bien qui constitue un modèle de comportement, tout en renvoyant aussi dans certains cas à une personne concrète, ${ }^{72}$ peut être assimilé à un standard. Un passage de Paul 34 Ad edictum D 192 24pr $r^{73}$ mentionne la bona fides et le vir bonus:

Si in lege locationis comprehensum sit, ut arbitratu domini opus adprobetur, perinde habetur, ac si viri boni arbitrium comprehensum fuisset, idemque servatur, si alterius cuiuslibet arbitrium comprehensum sit: nam fides bona exigit, ut arbitrium tale praestetur, quale viro bono convenit. $[\ldots]^{74}$

Ce texte ${ }^{75}$ porte sur une hypothèse de probatio operis, à savoir un mécanisme dont la fonction est de vérifier la qualité et la conformité au contrat de louage d'ouvrage d'un opus. Une clause du contrat de locatio conductio operis prévoyait que ce contrôle de conformité devait être fait arbitratu domini, c'est-à-dire selon le jugement du maître de l'ouvrage. Or, Paul requalifie l'arbitratus domini en arbitratus boni viri. Partant, le juriste sévérien transforme l'arbitratus du maître en un standard objectif d'homme de bien. Paul justifie cette requalification en invoquant les exigences qui découlent de la nature du contrat de bonne foi. Le jugement personnel du dominus qui n'a rien d'une décision arbitraire, ${ }^{76}$ contraste avec la fides bona. Cette incompatibilité s'explique probablement par le fait que la décision selon le jugement du maître de l'ouvrage ne répond pas à l'objectivité qui découle du standard de la bonne foi.

Tout comme la fides bona, l'arbitratus boni viri doit être regardé comme un modèle de comportement qui permet l'interprétation des actes juridiques. Ce standard présente un domaine d'application assez vaste et, comme la bonne foi, son emploi permet d'accroître les pouvoirs d'appréciation du juge. L'insertion du standard du jugement de l'homme de bien dans plusieurs formules des stipulations prétoriennes illustre parfaitement son utilité. La stipulatio praetoria est imposée par le préteur pour obliger une personne à tenir un certain comportement sous peine de sanctions. ${ }^{77}$ Par exemple, en matière d'usufruit, le préteur exige que l'usufruitier prête la cautio

72 C'est par exemple le cas de Neratius 5 ep D 172 76, 78, 80.

73 Ce texte a fait l'objet de l'analyse radicale d'Albertario 1924: à la page 300, mais cette critique souffre des excès de la doctrine interpolationniste.

74 "Si dans une clause de louage, il est prévu que l'approbation de l'ouvrage sera faite conformément au jugement du maître de l'ouvrage, cette disposition devra être interprétée comme si elle prévoyait le jugement d'un homme de bien. Cela s'appliquera même dans l'hypothèse où la clause ferait référence à n'importe quelle autre personne: en effet, la bonne foi impose que le jugement rendu soit tel qu'il serait convenable pour un homme de bien. Et ce jugement concerne la qualité de l'ouvrage et non pas la faculté de proroger le laps de temps dans lequel il doit être achevé selon la clause, à moins que cela ne soit pas compris dans la clause. De cela découle que l'approbation caractérisée par le dol du maître d'œuvre est nulle et il est possible d'agir sur la base de l'action de louage."

75 Cannata 1969: à la page 197; Voci 1969: à la page 201; Gallo 1970: aux pages 496sq; Martin 1989: aux pages 109sq.

76 À ce propos, voir Labeo 5 post a Iav epit D 192603.

77 Sur les stipulations prétoriennes, voir Palermo 1942; Mozzillo 1960; idem 1971: aux pages 450sq; Finkenauer 2010: aux pages 294sq. 
fructuaria en s'engageant à restituer le bien à la fin de l'usufruit, à utiliser et à jouir de ce bien conformément au jugement d'un homme de bien et à s'abstenir de commettre un dol. ${ }^{78}$ La création de la cautio fructuaria trouve son origine dans une lacune du ius civile qui ne sanctionnait pas les comportements négligents du fructuarius, comme c'est le cas de l'usufruitier qui ne taillait pas les vignes ou qui laissait aller en ruine l'aqueduc qui traversait le fonds. ${ }^{79}$ Grâce à l'intervention du préteur, le fructurius peut désormais être assigné en justice sur la base de l'actio ex stipulatu qui découle de la cautio fructuaria par le nu-propriétaire qui lui reproche de ne pas uti frui arbitratu boni viri.

En dépit de l'opinion contraire d'une partie de la doctrine ${ }^{80}$ ce renvoi au jugement d'un homme de bien ne doit pas être compris dans un sens subjectif. Il ne s'agit pas d'un tiers qui est appelé en tant qu'arbitre à apprécier si le comportement de l'usufruitier est en accord avec la stipulation. En effet l'analyse des nombreuses sources relatives à la cautio fructuaria ne permet pas de déduire l'intervention d'un tiers. Dès lors, l'arbitratus boni viri correspond à un standard. Il reviendra au iudex de vérifier que l'usufruitier a respecté le modèle du jugement de l'homme de bien qui est inclus dans la formule de la stipulation prétorienne. Le renvoi au jugement de l'homme de bien a des conséquences pratiques extrêmement importantes car il permet d'augmenter les pouvoirs d'appréciation du juge.

La mention de l'arbitratus boni viri autorise le juge à examiner les circonstances factuelles de l'affaire ce qui rend l'actio ex stipulatu, qui est une action de droit strict, plus flexible. L'inclusion du jugement de l'homme de bien peut donc être comparée à la clause ex fide bona. Néanmoins, l'actio ex stipulatu qui découle de la cautio fructuaria ne change pas pour autant sa nature. Elle reste une action stricti iuris. Il ne s'agit pas d'une contradiction car la peine encourue par l'usufruitier est établie par la stipulation et que le juge n'a aucune marge discrétionnaire pour la moduler. ${ }^{81}$ L'arbitratus boni viri constitue donc un standard, un modèle objectif qui s'impose au juge en lui donnant ainsi un pouvoir discrétionnaire. En même temps, le renvoi au jugement de l'homme de bien, par son caractère objectif, implique que le juge ne peut pas décider en se basant sur ses propres critères personnels, mais il doit tenir compte du standard du vir bonus qui implique nécessairement une idée de bon sens et de modération.

78 Lenel $1927^{3}$ : aux pages 538-539, qui est suivi par Mantovani 1999²: à la page 106, a ainsi reconstruit la formule de la cautio fructuaria: "Cuius rei usus fructus testamento Lucii Titii tibi legatus est, ea re boni viri arbitratu usurum fruiturum te et, cum usus fructus ad te pertinere desinet, id quod inde extabit restiturum iri dolumque malum abesse afuturumque esse spondesne? Spondeo." ("Promets-tu d'utiliser et de jouir de cette chose, qui t'a été laissée par legs dans le testament de Lucius Titius, conformément au jugement d'un homme de bien, et quand l'usufruit ne t'appartiendra plus, de restituer ce qui restera et de t'abstenir, même dans le futur, du dol? Je promets.").

79 Ulpien 18 ad Sab D 71132.

80 Cannata 2001: aux pages 484-486; Santucci 2013: aux pages 139sq.

81 Dans ce sens, Betancourt 1973: à la page 381; Naumowicz 2011: à la page 603. 
Le modèle de l'homme de bien, tout en étant présent dans des nombreux domaines du droit romain, ne devient pas une ratio qui motive une certaine solution juridique. ${ }^{82}$ Dans ce sens, il est nécessaire de le distinguer du bonum et aequum qui dans certaines hypothèses ne doit pas être assimilé à un standard.

S'agissant des stipulations prétoriennes, le renvoi au jugement de l'homme de bien apparaît à plusieurs reprises dans la formule. Mais parfois l'application du standard de l'arbitratus boni viri se fait en dehors de toute mention formulaire. L'analyse de plusieurs fragments relatifs aux successions révèle ainsi comment les jurisconsultes ont recours à l'homme de bien pour compléter des dispositions à cause de mort. Il en va ainsi d'un célèbre passage de Celse 15 Digesta D 32 43:83

Si filiae pater dotem arbitratu tutorum dari iussisset, Tubero perinde hoc habendum ait ac si viri boni arbitratu legatum sit. Labeo quaerit, quemadmodum apparet, quantam dotem cuiusque filiae boni viri arbitratu constitui oportet: ait id non esse difficile ex dignitate, ex facultatibus, ex numero liberorum testamentum facientis aestimare. ${ }^{84}$

Dans une hypothèse de legs de dot dont le montant est indéterminé, Tubéron affirme que la somme doit être évaluée conformément au jugement d'un homme de bien. Cette solution est approuvée par Labéon qui l'approfondie en se demandant en quoi consiste exactement l'arbitratus boni viri. Ce dernier montre comment appliquer dans ce cas ce standard. Il s'agit de la prise en compte des données objectives, à savoir le rang et le patrimoine du testateur, le nombre d'enfants qu'il laisse.

Ce texte est tout à fait remarquable car il donne des exemples précis de paramètres que le vir bonus romain aurait pris en compte pour calculer la dot de sa fille. De surcroît, le testateur transmet la dot par le biais d'un legs. Or, le legatum à effets obligatoires est protégé par l'actio ex testamenti qui est une action de droit strict. En dépit de cela, les jurisconsultes emploient le standard de l'homme de bien tout comme ils le font en matière de fidéicommis - une institution qui comme l'indique sa dénomination même, a des liens extrêmement étroits avec la fides. Même si les textes qui portent sur les fideicommissa sont plus nombreux, ${ }^{85}$ l'application par la jurisprudence du standard de l'homme de bien dans un acte juridique protégé par une action de droit strict montre l'ampleur pratique de la technique du standard. Il ne reste maintenant qu'à comprendre pour quelle raison les prudentes y ont recours.

82 Une intéressante exception est néanmoins constituée par Papinien 27 quaest D 18761.

83 Astolfi 1964; Grosso 1966³ à la page 138; Voci 1963: à la page 882; idem 1969: à la page 221; Solinas: 1972 à la page 560; Gallo 1971: à la page 582; Stagl 2009: à la page 131.

84 "Si un père a laissé à sa fille une dot conformément au jugement des tuteurs, Tubéron dit qu'il faut considérer que la dot a été léguée conformément au jugement d'un homme de bien. Labéon demande comment déterminer le montant de la dot qui doit être constituée en faveur de la fille conformément au jugement d'un homme de bien. Il dit que cela n'est pas difficile à estimer sur la base de la position sociale, du patrimoine et du nombre d'enfants du testateur."

85 Par exemple, Scaevola 4 resp D 33113 1; Papinien 9 resp D 34110 2; Modestin 11 resp D 341 5. 
La disposition testamentaire mentionnée dans le fragment de Celse D 3243 est indéterminée et, de la sorte, destinée à l'inefficacité. Or, seul l'interprète par son œuvre herméneutique peut reconstruire la volonté du de cuius en permettant ainsi l'application du legs. Pour atteindre ce résultat, le jurisconsulte emploie le modèle du vir bonus, en calculant le montant de la dot comme le ferait un homme de bien, une personne honnête.

Le vir bonus renvoie au modèle de l'homme honnête, ce qui, à première vue, pourrait sembler être proche de celui du bonus ou diligens pater familias. Toutefois, cette proximité est seulement apparente: il s'agit de deux standards distincts. En effet, le bon ou diligent père de famille est le standard qui permet exclusivement l'appréciation de la culpa. ${ }^{86}$

\section{Le bonus ou diligens pater familias}

Une illustration de l'emploi du bonus ou diligens pater familias se trouve dans un passage de Labéon, extrait du livre 2 des Pithana épitomé par Paul au Digeste 191 $54 p r:$

Si servus quem vendideras iussu tuo aliquid fecit et ex eo crus fregit, ita demum ea res tuo periculo non est, si id imperasti, quod solebat ante venditionem facere, et si id imperasti, quod etiam non vendito servo imperaturus eras. Paulus: minime: nam si periculosam rem ante venditionem facere solitus est, culpa tua id factum esse videbitur: puta enim eum fuisse servum, qui per catadromum descendere aut in cloacam demitti solitus esset. Idem iuris erit, si eam rem imperare solitus fueris, quam prudens et diligens pater familias imperaturus ei servo non fuerit. Quid si hoc exceptum fuerit? Tamen potest ei servo novam rem imperare, quam imperaturus non fuisset, si non venisset: veluti si ei imperasti, ut ad emptorem iret, qui peregre esset: nam certe ea res tuo periculo esse non debet. Itaque tota ea res ad dolum malum dumtaxat et culpam venditoris dirigenda est. ${ }^{87}$

Ce texte ${ }^{88}$ porte sur une hypothèse spécifique de responsabilité dans la vente qui se pose quand le venditor n'a pas encore transmis la possession de l'esclave objet

86 Voir Cannata 1992: aux pages 28-29.

87 "Si l'esclave que tu as vendu sur ton ordre a fait quelque chose et à cause de cela il s'est cassé une jambe, cette chose n'est pas faite à ton péril seulement si tu as ordonné ce qu'il avait l'habitude de faire avant la vente, et si tu as ordonné ce que tu aurais ordonné à une esclave que tu n'avais pas vendu. Paulus: Pas du tout. En effet, s'il avait l'habitude de faire une chose dangereuse avant la vente, il semblera que cela a été fait de ta faute: imagine en effet un esclave qui a l'habitude de descendre à la corde ou qui se laisse baisser dans les égouts. La même règle de droit s'applique s'il avait l'habitude d'ordonner une chose qu'un prudent et diligent père de famille n'aurait pas ordonnée à cet esclave. Qu'est-ce qu'il arrive si cela a été excepté expressément? Il peut certes ordonner une nouvelle chose à cet esclave qu'il n'aurait pas ordonné s'il ne le vendait pas: par exemple, si tu lui as ordonné d'aller chez l'acheteur qui est à l'étranger. En effet, cela ne doit certainement pas être à ton péril. En effet, toute cette affaire doit être seulement déterminée sur la base du dol et de la faute du vendeur." 
de la vente. Dans ce laps de temps avant la traditio, l'esclave se casse une jambe en exécutant une activité qui lui a été ordonnée par le vendeur. Partant, le vendeur est-il responsable de l'accident qui est survenu? Le texte présente deux solutions différentes.

Labéon exclut la culpa du vendeur si l'esclave s'est blessé en accomplissant une activité qu'il avait l'habitude de faire même avant la vente. Il est possible que

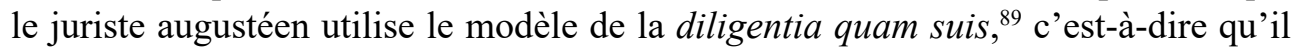
fasse référence à la diligence que le pater familias utilisait à l'égard de choses qui lui appartiennent. Le critère de la diligentia quam suis demeure difficilement identifiable à la technique du standard car les juristes apprécient la responsabilité sur la base du comportement qu'une personne adopte avec ses biens personnels. Il manque alors ce profil de l'indétermination, qui est typique du standard. En effet, s'il est bien question de normalité, c'est-à-dire la manière dans laquelle le pater familias a l'habitude de traiter ses biens, le profil de l'indétermination qui est typique de la technique du standard s'affaiblit fortement car les jurisconsultes prennent en considération l'homme dont il faut établir la culpa. Il s'agit dès lors de l'appréciation

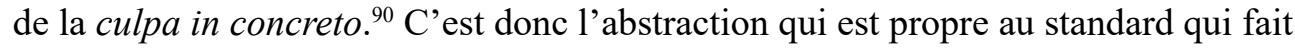
ici défaut. ${ }^{91}$

88 La partie finale du texte, à partir de quod si exceptum fuerit, a fait l'objet de soupçons d'interpolations. La tendance est aujourd'hui de considérer le contenu du fragment comme authentique. Voir Voci 2010: à la page 84; Cannata 1992: à la page 40.

89 Cannata 1992: à la page 40. Sulla diligentia quam suis, voir Maganzani 2006; Santucci 2008.

90 Le texte probablement plus célèbre qui porte sur une hypothèse de diligentia quam suis est un fragment du livre 11 des Digesta de Celse transmis au Digeste 163 32, qui a été connu à partir du Moyen Âge comme lex quod Nerva. Ce fragment est connu aussi pour l'assimilation entre la culpa lata et le dol opérée par Nerva. Sur ce texte voir notamment, Maganzani 2006: passim.

91 De manière analogue, il semble impossible d'assimiler à un standard la simple mention du pater familias, comme c'est le cas dans le fragment d'Ulpien 17 Ad Sabinum D 719 7: "Instrumenti autem fructum habere debet: vendendi tamen facultatem non habet. Nam et si fundi usus fructus fuerit legatus et sit ager, unde palo in fundum, cuius usus fructus legatus est, solebat pater familias uti, vel salice vel harundine, puto fructuarius hactenus uti posse, nec ex eo vendat, nisi forte salicti ei vel silvae palaris vel harundineti usus fructus sit legatus: tunc enim et vendere potest. Nam et Trebatius scribit silvam caeduam et harundinetum posse fructuarium caedere, sicut pater familias caedebat, et vendere, licet pater familias non solebat vendere, sed ipse uti: ad modum enim referendum est, non ad qualitatem utendi." ("L'usufruitier doit avoir le fruit de ce qui sert à l'exploitation du fonds, toutefois il n'a pas la faculté de le vendre. En effet, même si on laisse par legs l'usufruit d'un fonds et qu'il y ait un champ, où le père de famille avait l'habitude de prendre les pieux, les saules et les joncs pour le fonds, dont on a laissé l'usufruit, je considère que l'usufruitier peut les utiliser, avec la limite qu'il ne peut rien en vendre, à moins qu'on ne lui ait laissé l'usufruit du bois des saules, du bois pour les pieux ou de la cannaie. Dans ce cas, il peut les vendre. En effet, Trebatius aussi écrit que l'usufruitier peut couper le bois taillis ou la cannaie, comme le couperait le père de famille, et vendre, même si le père de famille n'avait pas l'habitude de vendre, mais de les utiliser lui-même. Il faut en effet avoir égard à la façon de l'utilisation et non pas à sa qualité.") Dans cette hypothèse, le juriste fait référence aux habitudes du propriétaire du fonds pour déterminer l'ampleur de l'exploitation de l'usufruitier. 
Pour déterminer l'existence d'une culpa, Paul applique en revanche un modèle objectif, celui du père de famille prudent et diligent. Les activités habituelles de l'esclave ne rentrent donc pas en ligne de compte pour apprécier la responsabilité du vendeur. Paul admet toutefois l'existence d'accords entre les deux parties qui peuvent moduler la responsabilité du vendeur. Dans ce cas, le vendeur pourra ordonner au servus d'accomplir des activités qu'il n'aurait jamais faites s'il n'avait pas été vendu.

Selon Paul la culpa s'apprécie sur la base du standard du diligens et prudens pater familias. Ce modèle correspond à celui du bonus pater familias car les jurisconsultes romains emploient de nombreuses expressions équivalentes pour renvoyer au même standard..$^{92}$ Il faut en effet rappeler que si le Code civil français a choisi de faire référence au bon père de famille, cette formulation du modèle n'était probablement pas la plus répandue à Rome. Cela se déduit du fait que seules cinq occurrences du renvoi au bon père de famille dans le Digeste ont survécu. ${ }^{93}$

Il reste à expliquer la signification du standard: quelle valeur peut-on attribuer à l'expression bonus ou diligens pater familias? Tout d'abord, il est nécessaire de souligner que le pater familias romain ne correspond pas au père de famille français. En effet, par le terme pater familias les Romains indiquent l'homme qui était sui iuris. Par conséquent, un homme qui n'est pas marié ou qui n'a pas d'enfants devient pater familias dès lors qu'il n'est pas in potestate d'un ascendant. Seuls les sui iuris jouissent de la capacité juridique en droit privé ${ }^{94}$ et peuvent avoir un patrimoine. En ce qui concerne l'adjectif diligens, il s'agit de la personne qui se comporte avec soin et application. La diligentia est dès lors la caractéristique de la personne qui agit avec scrupule et zèle. ${ }^{95}$ Le diligens pater familias est l'homme, doté de capacité juridique, qui se comporte avec vigilance et précaution. En revanche, le terme bonus a une signification bien plus large par rapport à diligens. Pour cette raison, il a fait l'objet d'interprétations très différentes de la part de la doctrine romanistique. ${ }^{96}$ Sans entrer dans les détails de cette controverse, on peut se limiter à affirmer que bonus a un champ sémantique assez vaste, ${ }^{97}$ mais de manière générale il renvoie à l'idée de diligence chez un homme et de valeur pour une chose. ${ }^{98}$ Cette signification de bonus, proche du mot diligens, explique dès lors pour quelle raison les jurisconsultes emploient de manière équivalente le standard du bon et diligent père de famille.

92 Sur ce point, voir Cannata 1992: à la page 29 note 95.

93 Ulp 17 ad Sab D 719 2; Paul 3 ad Sab D 7815 1; Gai 10 ad ed prov D 18135 4; Paul 40 ad ed. D 381201 (bonus et diligens); Afr 9 quaest D 40422.

94 Sur les conflits qu'une telle situation peut engendrer, voir Thomas 1982: aux pages 5sq.

95 Voir Ernout \& Meillet $1967^{4}$ : à la page 350.

96 On fait notamment allusion à la querelle qui a opposé récemment deux romanistes italiens, Giuseppe Falcone et Roberto Fiori. Voir notamment Falcone 2010-2011: aux pages 57sq [= idem 2013a: aux pages 39sq]; idem 2014: aux pages 258sq. Fiori 2011b; idem 2013a: aux pages 19sq; idem 2013b: aux pages 169sq.

97 Ernout \& Meillet 19674: à la page 73.

98 De Meo 1986²: à la page 212. 
Une autre belle illustration de l'application de la technique du standard pour l'appréciation de la responsabilité se trouve dans un passage de Gaius, qui est extrait du livre 10 de son commentaire Ad edictum provinciale, au Digeste 19225 7:

\begin{abstract}
Qui columnam transportandam conduxit, si ea, dum tollitur aut portatur aut reponitur, fracta sit, ita id periculum praestat, si qua ipsius eorumque, quorum opera uteretur, culpa acciderit: culpa autem abest, si omnia facta sunt, quae diligentissimus quisque observaturus fuisset. Idem scilicet intellegemus et si dolia vel tignum transportandum aliquis conduxerit: idemque etiam ad ceteras res transferri potest. ${ }^{99}$
\end{abstract}

Ce texte de Gaius est très célèbre. Il a fait l'objet d'une attention considérable de la part de la doctrine ${ }^{100}$ et a surtout donné lieu à de nombreuses critiques sur son éventuelle interpolation. ${ }^{101} \mathrm{Il}$ est ici question d'une locatio conductio qui a pour objet le transport d'une colonne. Le conductor est-il responsable si la colonne se casse? La réponse de Gaius est claire: la responsabilité du transporteur peut être engagée seulement si une faute a été commise par lui ou par une personne qui travaille sous ses ordres. ${ }^{102}$ L'absence de culpa est dès lors définie par le jurisconsulte comme le fait d'avoir accompli tout ce qu'aurait fait un homme très diligent ("si omnia facta sunt, quae diligentissimus quisque observaturus fuisset"). L'on constate que le standard est analogue au bonus ou diligens pater familias, mais il n'est pas identique car il est question d'un diligentissimus quisque. Faut-il attacher de l'importance à cette différence? L'emploi de l'adjectif au superlatif peut-il être considéré comme un indice d'interpolation?

Rien n'autorise cependant à penser que diligentissimus quisque est un ajout des compilateurs. ${ }^{103}$ En dépit de cette différence dans la formulation, l'emploi du superlatif ne semble pas impliquer l'existence d'une différence conceptuelle. L'emploi de diligentissimus est simplement une expression particulière du modèle de l'homme diligent. Comme il a été souligné plus haut, les sources romaines ont recours à plusieurs formulations pour indiquer le standard qui permet l'appréciation

99 “La personne qui s'est engagé par un contrat de louage à transporter une colonne, si elle, pendant qu'il la soulève ou la transporte ou la replace, est cassée, cette personne est responsable pour le risque seulement si cela arrive pour sa propre faute ou pour la faute de ceux dont elle sert de l'œuvre. En revanche, il n'y a pas de faute si tout ce qu'observerait une personne très diligente a été fait. Évidemment, nous comprendrons de la même manière si quelqu'un s'est engagé par un contrat de louage à transporter des jarres ou une poutre. Et cette règle peut être aussi appliquée à d'autres situations."

100 On se limitera à citer quelques-unes des nombreuses contributions: Alzon 1966: à la page 328; MacCormack 1971: à la page 541; idem 1972: aux pages 137 et 170; Robaye 1987: praecipue à la page 206; Voci 1990: aux pages 38, 85, 106, 132; Cannata 1992: praecipue aux pages 38, 44.

101 À ce propos, voir Robaye 1987: aux pages 206sq.

102 Fercia 2008.

103 Cardilli 1995: à la page 500. Il existe des analogies avec deux autres passages (Gai 9 ad ed prov D 136 18pr; idem, 10 ad ed prov D 18135 4), ce qui constitue une preuve importante du caractère classique de D 192257. 
de la culpa. En l'espèce, l'utilisation du superlatif s'explique probablement par la nécessité de caractériser la faute dont répond le débiteur. ${ }^{104}$ Quisque diligentissimus serait donc une simple déclinaison du bonus ou diligens pater familias.

\section{Conclusion}

Le concept de standard est très récent puisqu'il a fait son apparition dans le langage juridique au début du $\mathrm{xx}^{\mathrm{e}}$ siècle. En dépit du caractère contemporain du terme, il est possible d'analyser certaines clauses romaines sous l'angle de standard. En effet, l'analyse des sources juridiques romaines a révélé que si le concept de standard n'a pas été théorisé par la iurisprudentia, il existe néanmoins plusieurs critères herméneutiques qui correspondent de facto à des standards, comme la fides bona, le vir bonus ou les boni mores. Tout en ayant conscience de l'anachronisme, il paraît fécond d'utiliser le terme standard pour analyser les sources romaines. Cette catégorie juridique constitue un outil très efficace pour appréhender les textes romains puisqu'il permet d'identifier des lignes de force et de comprendre pour quels raisons les romains ont utilisé ces expressions que l'on a identifié comme des standards.

Leur emploi a été de grande importance puisqu'il a permis d'élargir, même dans le domaine des actions de droit strict, le pouvoir d'appréciation du juge. Le standard est dès lors un outil fondamental qui permet, par le truchement du juge, d'adapter le droit aux exigences de la conscience sociale.

\section{ABSTRACT}

It is difficult to develop a definition of the legal concept of standard. In spite of this the term "standard" is often used in French law. For example, good faith (bonne foi), sound morals (bonnes mours) and pater familias (bon père de famille) are considered standards. We find the same concepts in Roman law. Moreover, it appears that the use of this kind of concepts is precocious: the action of the fiducia, which dates back at least to the second century $\mathrm{BC}$, mentions the clause ut inter bonos bene agier. Yet, as is often the case, there is no general category that encompasses all these concepts. It is consequently tempting to classify them as "standards" to fill this void. Methodologically speaking, however, it is hard to establish if it is correct to use the term "standard". In fact, this classification raises a problem of coherence. The legal use of the term "standard", which is of Anglo-Saxon origin, is recent. It dates back to the 1920s. N Roscoe Pound probably used it first and had an important role in its success. French lawyers began to employ the concept of "standard" to achieve a more flexible interpretation of the statutory law. Is it pertinent to use a clearly anachronistic word to describe Roman reality? It would be reasonable to fear

104 Voir la réflexion de Cardilli 1995: aux pages 496sq sur le diligentissimus pater familias présent dans Gai, 9 ad ed prov D 136 18pr. 
that the application of the term "standard" would result in a deformation of Roman law. Though, an analysis of the sources shows that it is possible and even fruitful to apply the contemporary concept of "standard" to Roman law. While it is true that it is anachronistic, using it seems to improve our understanding of Roman law.

\section{BIBLIOGRAPHY}

Albertario, E (1924) L'arbitrium boni viri del debitore nella determinazione della prestazione (Milano)

Al-Sanhouri, A (1925) Les restrictions contractuelles à la liberté du travail dans la jurisprudence anglaise: Contribution à l'étude comparative de la règle de droit et du standard juridique (Paris)

Alzon, C (1966) “Les risques dans la 'locatio-conductio"' Labeo 12: 311-337

Astolfi, R (1964) Studi sull'oggetto dei legati in diritto romano vol 1 (Padova)

Behrends, O (2002) "Dalla mediazione arbitrale alla protezione giudiziaria. Delle formule di buona fede e delle cd. formule in factum conceptae" in Cascione C et al (éds) Diritto e giustizia nel processo. Prospettive storiche, costituzionali e comparatistiche (Napoli): 197323

Bernard, E (2010) La spécificité du standard juridique en droit communautaire (Bruxelles)

Bertoldi, F (2012) Il negozio fiduciario nel diritto romano classico (Modena)

Beseler, G (1925) “Miszellen: [Aequitas]” Zeitschrift der Savigny Stiftung für RechtsgeschichteRomanistische Abteilung 45: 396-488

Betancourt, F (1973) “Sobre una pretendida 'actio' arbitraria contra el usufructuario" Anuario de historia del derecho español 43: 353-383

Betti, E $\left(1997^{2}\right)$ "Diritto romano e dogmatica odierna" in De Francisci, P Questioni di metodo. Diritto romano e dogmatica odierna (Como): 25-83

Biscardi, A (1973) "Riflessioni minime sul concetto di "aequitas" in Studi in memoria di Guido Donatuti vol 1 (Milano): 137-142

Blanc, N (2016) "Le juge et les standards juridiques" Revue des contrats 2: 394-397

Buckland, WW (1930) "Diligens pater familias" in Studi in onore di Pietro Bonfante nel XL anno del suo insegnamento vol 2 (Milano): 85-108

Cannata, CA (1969) Per lo studio della responsabilità per colpa. Corso di diritto romano nell'Università di Cagliari anno accademico 1967-1968 (Milano)

Cannata, CA (1992) "Sul problema della responsabilità del diritto privato romano" IVRA 43: 1-82

Cannata, CA (2001) Corso di istituzioni di diritto romano vol 1 (Torino)

Carbonnier, J (1984) "Les notions à contenu variable dans le droit français de la famille" in Perelman, C \& Vander Elst, R Les notions à contenu variable en droit (Bruxelles): 99-112

Cardilli, R (1995) L'obbligazione di "praestare” e la responsabilità contrattuale in diritto romano (II sec. a. C. - II sec. d. C.) (Milano)

Cardilli, R (2010²) "Bona fides" tra storia e sistema (Torino)

Dale, W (1988) “The use of standards in the English legal system” Revue de la recherche juridique, droit prospectif 4: $887-894$ 
De Francisci, P (1936) "Questioni di metodo" in Studi in onore di Salvatore Riccobono vol 1 (Palermo): 1-19 = De Francisci, P (19972) "Questioni di metodo" Questioni di metodo. Diritto romano e dogmatica odierna (Como): 85-104

De Loynes de Fumichon, B (2012) "Le bénéfice de compétence, une exceptio pétrie de bons sentiments" in Chevreau, E, Kremer, D \& Laqurrière-Lacroix, A Carmina iuris. Mélanges en l'honneur de Michel Humbert (Paris): 495-522

De Meo, C (1986²) Lingue tecniche del latino (Bologna)

Delebecque, P (1988) "Les standards dans les droit romano-germaniques" Revue de la recherche juridique, droit prospectif 4: 871-885

Delmas-Marty, M (1988) "Les standards en droit pénal" Revue de la recherche juridique, droit prospectif 4: 1019-1035

Dunand, J-P (2000) Le transfert fiduciaire: “donner pour reprendre”. Mancipio dare ut remancipetur. Analyse historique et comparatiste de la fiducie-gestion (Bâle-GenèveMunich)

Ernout, A \& Meillet, A (1967 ) Dictionnaire étymologique de la langue latine. Histoire des mots (Paris)

Falcone, G (2010-2011) “L'attribuzione della qualifica 'vir bonus' nella prassi giudiziaria di età repubblicana (a proposito di Cato, or. fr. 186 Sblend.= 206 (Malc.)" Annali del Semninario giuridico dell'Università di Palermo 54: 57-93 [= Falcone, G (2013a) "L'attribuzione della qualifica 'vir bonus' nella prassi giudiziaria d'età repubblicana. Con un'appendice su 'optimus', 'probus', ‘fortis'” Lovato, A Vir bonus. Un modello ermeneutico della riflessione giuridica antica (Incontro di studio, Trani, 28-29 ottobre 2011) (Bari): 39-90]

Falcone, G (2013) "Il rapporto ius gentium - ius civile e la societas vitae in Cic., off. 3.69-70" Annali del Seminario giuridico dell'Università di Palermo 56: 259-274

Falcone, G (2014) "La formula 'ut inter bonos bene agier oportet et sine fraudatione' e la nozione di "vir bonus"" Meditationes de Iure et Historia. Essays in Honour of Laurens Winkel, Fundamina 20-1: 258-274

Fercia, R (2008) La responsabilità per fatto di ausiliari nel diritto romano (Padova)

Finkenauer, Th (2010) Vererblichkeit und Drittwirkungen der Stipulation im klassischen römischen Recht (Tübingen)

Fiori, R (1998-1999) "Ius civile, ius gentium, ius honorarium: il problema della 'recezione' dei iudicia bonae fidei" Bullettino dell'Istituto di diritto romano "Vittorio Scialoja” 101102: $165-197$

Fiori, R (2008) "Fides e bona fides. Gerarchia sociale e categorie giuridiche" Modelli teorici e metodologici nella storia del diritto privato vol 3 (Napoli): 237-259

Fiori, R (2011a) "Bona fides. Formazione, esecuzione e interpretazione del contratto nella tradizione civilistica (Seconda parte)" Modelli teorici e metodologici nella storia del diritto privato vol 4 (Napoli): 97-242

Fiori, R (2011b) Bonus vir. Politica, filosofia e retorica nel De Officiis di Cicerone (Napoli)

Fiori, R (2013a) "Il vir bonus tra filosofia greca e tradizioni romane nel de officiis di Cicerone" Lovato, A Vir bonus. Un modello ermeneutico della riflessione giuridica antica (Incontro di studio, Trani, 28-29 ottobre 2011) (Bari): 19-38

Fiori, R (2013b) "La gerarchia come criterio di verità: 'boni' e 'mali' nel processo romano arcaico" in Cascione, C et al (éds) Quid est veritas? Un seminario su verità e forme giuridiche (Napoli): 169-250 


\section{L'EMPLOI DES STANDARDS EN DROIT ROMAIN}

Gallo, F (1970) La dottrina di Proculo e quella di Paolo in materia di arbitraggio, in Studi in onore di Giuseppe Grosso vol 3 (Torino): 477-542

Gallo, F (1971) "Nuovi spunti nell'interpretazione di C. 5.11.1" Studi in onore di Edoardo Volterra vol 4 (Milano): 573-599

Gallo, F (2000) "A proposito di 'aeque' in D. 19.1.50: un giudizio con comparazione sottesa" Studia et documenta historiae iuris 66: 1-27

Giannozzi, E (2015) Le vir bonus en droit romain (thèse dactylographiée, Université PanthéonAssas Paris II)

Grosso, G (1966 ${ }^{3}$ ) Obbligazioni. Contenuto e requisiti della prestazione (Torino)

Guarino, A (1960) “Equità (diritto romano)” Novissimo Digesto Italiano 6: 619-624

Josserand, L (1939²) De l'esprit des droits et de leur relativité (Paris)

Kunkel, W (1925) "Diligentia" Zeitschrift der Savigny Stiftung für RechtsgeschichteRomanistische Abteilung 45: 266-351

Lenel, O (19273) Das Edictum Perpetuum. Ein Versuch zu seiner Wiederherstellung (Leipzig)

Lévy, J-Ph (1937) Les impenses dotales en droit romain classique (Paris)

MacCormack, G (1971) "Culpa in eligendo" Revue internationale des droits de l'antiquité 18: $525-551$

MacCormack, G (1972) “Culpa” Studia et documenta historiae iuris 38: 123-188

MacCormick, N (1984) “On reasonableness” in Perelman, C \& Vander Elst, R Les notions à contenu variable en droit (Bruxelles): 131-156

Maganzani, L (2006) La "diligentia quam suis" del depositario dal diritto romano alle codificazioni nazionali (Milano)

Mantello, A (2007) La retorica di Celso figlio. A proposito d'una quaestio de bono et aequo in Studi per Giovanni Nicosia vol 5 (Milano): 121-142

Mantovani, D (1999²) Le formule del processo privato romano (Padova)

Martin, SD (1989) The Roman Jurists and the Organization of Private Building in the Late Republic and Early Empire (Bruxelles)

Maschi, CA (1962) "Certezza del diritto e potere discrezionale del magistrato nel diritto romano" in Studi in onore di Emilio Betti vol 3 (Milano): 411-449

Miceli, M (2008) Studi sulla "rappresentanza” nel diritto romano vol 1 (Milano)

Mozzillo, A (1960) Contributi allo studio delle "stipulationes praetoriae” (Napoli)

Mozzillo, A (1971) "Stipulatio praetoria” Novissimo Digesto italiano XVIII: 450-456

Navarro, J-S (1988) "Standards et règles de droit" Revue de la recherche juridique, droit prospectif 4: $833-845$

Naumowicz, P (2011) Fidei bonae nomen et societas vitae. Contribution à l'étude des actions de bonne foi (thèse dactylographiée, Université Panthéon-Assas Paris II)

Noordaven, B (1999) Die Fiduzia im Römischen Recht (Amsterdam)

Orianne, P (1988) "Les standards et les pouvoirs du juge" Revue de la recherche juridique, droit prospectif 4: 1037-1067

Ouedraogo, A (2013) "Standard et standardisation: la normativité variable en droit international" Revue québécoise de droit international 26(1): 155-186 


\section{ELENA GIANNOZZI}

Palermo, A (1942) Il procedimento cauzionale nel diritto romano (Milano)

Palma, A (1992) Humanior interpretatio, "Humanitas" nell'interpretazione e nella normazione da Adriano ai Severi (Torino)

Paricio, J (1986) Estudios sobra las actiones in aequum conceptae (Milano)

Pattaro, E (1988) "Les dimensions éthiques de la notion de standard juridique" Revue de la recherche juridique, droit prospectif 4: 813-823

Perelman, C \& Vander Elst, R (1984) Les notions à contenu variable en droit (Bruxelles)

Petev, V (1988) "Standards et principes généraux du droit” Revue de la recherche juridique, droit prospectif 4: $825-831$

Pringsheim, F (1932) “Bonum et aequum” Zeitschrift der Savigny Stiftung für RechtsgeschichteRomanistische Abteilung 52: 78-155

Rials, S (1980) Le juge administratif français et la technique du standard (essai sur le traitement juridictionnel de l'idée de normalité) (Paris)

Robaye, R (1987) L'obligation de garde. Essai sur la responsabilité contractuelle en droit romain (Bruxelles)

Roscoe Pound, N (1919) "The administrative application of legal standards." Allocution à la rencontre de l'American Bar Association (Boston)

Santucci, G (2008) Diligentia quam in suis (Trento)

Santucci, G (2013) "Il giudizio del vir bonus nel diritto di usufrutto" in Lovato, A Vir bonus. Un modello ermeneutico della riflessione giuridica antica (Incontro di studio, Trani, 28-29 ottobre 2011) (Bari): 139-160

Scevola, R (2008) “Negotium mixtum cum donatione'. Origini terminologiche e concettuali” (Padova)

Solinas, GP (1972) A proposito dell' "arbitrium boni viri” in Studi in onore di Gaetano Scherillo vol 2 (Milano): 539-571

Stagl, JF (2009) Favor dotis. Die privilegierung der Mitgift im System des römischen Recht (Wien-Köln-Weimar)

Stati, M (1927) Le standard juridique (Paris)

Sturlèse, B (2016) "Le juge et les standards juridiques" Revue des contrats 2: 398-399

Talamanca, M (1997) "Lex e interpretatio in Lab. 4 post. a Iav. Epit. D. 19.1.50" Nozione formazione e interpretazione del diritto. Dall'età romana alle esperienze moderne. Ricerche dedicate al Professor Filippo Gallo (Napoli): 353-439

Thomas, Y (1982) "La peur des pères et la violence des fils: images rhétoriques et normes du droit" Droit et cultures 4: 5-24

Varvaro, M (2006) Studi sulla restituzione della dote. I. La formula dell'actio rei uxoriae (Torino)

Viney, F (2013) Le bon père de famille et le plerumque fit. Contribution à l'étude des standards normatifs et descriptifs (thèse dactylographiée, Université Paris I Panthéon-Sorbonne, 2013)

Voci, P (1963) Diritto ereditario romano. Volume secondo. Parte speciale (Milano)

Voci, P (1969) Le obbligazioni romane (Corso di Pandette). Il contenuto dell'obligatio I, I (Milano) 


\section{L'EMPLOI DES STANDARDS EN DROIT ROMAIN}

Voci, P (1987) “Tradizione, donazione, vendita da Costantino a Giustiniano" IVRA 38: 72-148

Voci, P (1990) “'Diligentia', 'custodia', 'culpa'. I dati fondamentali” Studia et documenta historiae iuris 56: 29-143

Wroblewski, J (1988) "Les standards juridiques: problèmes théoriques de la législation et de

l'application du droit" Revue de la recherche juridique, droit prospectif 4: 847-869 